\title{
Micromagnetic Investigation of Microwave Permeability of Magnetic Artificial Spin Ice
}

\author{
Fusheng Ma, Yuping Wu, Baoyu Zong \\ Temasek Laboratories, National University of Singapore, Singapore, Singapore \\ Email: Fusheng.Ma@gmail.com
}

Received 27 September 2014; revised 26 October 2014; accepted 15 November 2014

Copyright (C) 2014 by authors and Scientific Research Publishing Inc.

This work is licensed under the Creative Commons Attribution International License (CC BY). http://creativecommons.org/licenses/by/4.0/

(c) (i) Open Access

\begin{abstract}
The complex permeability of the artificial spin ice array at different magnetic states is calculated using the micromagnetic simulation method. It is observed that the permeability spectra are dependent on the magnetization distributions of the array. The dependence of the permeability spectrum on the applied magnetic field strength and on the spacing gap between the neighboring elements is also investigated. Depending on the initial magnetization saturating direction, the permeability spectrum exhibits different sensitivity to the strength of the external applied magnetic field and to the spacing distance between the comprising elements of the artificial spin ice array.
\end{abstract}

\section{Keywords}

Microwave Permeability, Artificial Spin Ice, Micromagnetic Simulation, Magnetization Dynamics

\section{Introduction}

With the development of micro/nano-fabrication techniques, the exploration of nanometer-sized magnetic elements has markedly expanded in the recent years for their potential applications in advanced storage media, advanced microwave technologies, and novel spintronic devices. Recently, ferromagnetic frustration in "artificial spin ince" (ASI) was attracting intensive study [1]-[3]. ASIs are patterned nanoscale structures in which each element is small enough to have essentially uniform magnetization. The geometrical arrangement of the structures in the lattice leads to frustration by design to act as analogs of bulk geometrically frustrated materials [1] [4]. As these artificial structures are fabricated via nanolithography and thin film deposition, it is possible to engineer system parameters such as lattice geometry, inter-elemental dipolar coupling strength, moment switching behavior, and quenched disorder. In this work, we have calculated the microwave permeability of magnetic artificial spin ice consisting of stadium like elements on a square lattice using micromagnetic simulations. The 
complex permeability spectra of these artificial structures with different magnetic states are investigated. The permeability of ASIs at various magnetic field strength is also calculated, as well as their dependence on the spacing gap between the neighboring elements.

\section{Method}

The micromagnetic simulations are performed using a three-dimensional object-oriented micromagnetic framework (OOMMF) by solving the Landau-Lifshitz-Gilbert (LLG) equation as a function of time [5]. The microwave permeability spectra are obtained following the field-pulse processes [6]-[9]. The stable ground state of the magnetizations was firstly obtained by minimizing the total energy. Then, using the ground state as the initial state, the susceptibility is obtained by exciting the system with a small external field having a time dependence $h(t)=7.96 \times \exp (-7.675 t) \mathrm{A} / \mathrm{m}$ ( $t$ in ns), which is applied along the $y$ axis as shown in Figure 1(a). The Fourier transform of this expression leads to $h(\omega)=7.96 /(7.675+2 \pi \omega i)$ ( $\omega$ in GHz). This form has a significant response up to $20 \mathrm{GHz}$, which enables us to calculate the susceptibility up to this frequency. This field is spatially homogeneous and is introduced in the density functional of the Zeeman term. The amplitude of the driving field is very small to minimize nonlinear behaviour. We define the spatial average susceptibility in the direction $u$ as

$$
\langle M(t)\rangle \cdot u=\int_{-\infty}^{+\infty} \chi\left(t-t^{\prime}\right)[h(t) \cdot u] \mathrm{d} t^{\prime}
$$

where

$$
\langle M(t)\rangle=\frac{1}{V} \int M(r, t) \mathrm{d} r
$$

Performing FFTs on the excitation and the response, the obtained expression of the susceptibility can be directly calculated:

$$
M(t)=(\chi \star h)(t) \leftrightarrow M(\omega)=\chi(\omega) \cdot h(\omega)
$$

Based on the calculated dynamic susceptibility $\chi(\omega)$, the microwave permeability $\mu(\omega)$ can be derivate as $\mu(\omega)=\chi(\omega)+1$. The material parameters in OOMMF for Permalloy (Py) are taken: saturation magnetization $M_{s}=8.6 \times 10^{5} \mathrm{~A} / \mathrm{m}$, exchange stiffness constant $\mathrm{A}=13 \times 10^{-12} \mathrm{~J} / \mathrm{m}$. The gyromagnetic ratio $\gamma$ is set to be $2.21 \times 10^{5} \mathrm{~mA}^{-1} \mathrm{~S}^{-1}$ and the damping constant $\alpha$ is set to be 0.015 . A cubic cell size of $4 \times 4 \times 4 \mathrm{~nm}^{3}$ is used during the simulation. The investigated artificial square spin ice array consists 112 Pyelements, which distribute on a square lattice as shown in Figure 1(a), and each element (stadium) is of the length of $L=290 \mathrm{~nm}$ long, of the width $W=130 \mathrm{~nm}$ wide, of the thickness $20 \mathrm{~nm}$, and the gap between neighboring elements $G$ as shown in Figure 1(b). To investigate the effect of the gap $G$ on the microwave permeability, various values of $G$ are studied.

\section{Results and Discussion}

The magnetic states of the ASI lattice are prepared by saturating the array along the (10) and the (11) directions, respectively, and then gradually decrease the external field to certain value to realize the minimization of the total energy. Two kinds of stable states of the ASI lattice RS10 and RS11 are achieved by saturating the array along the (10) and (11) directions and gradually reducing the external field to fixed value, respectively. The

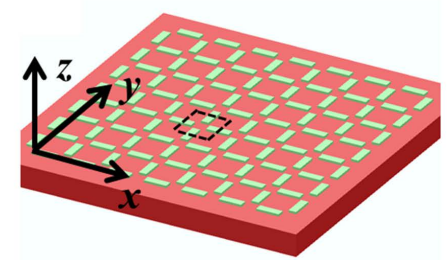

(a)

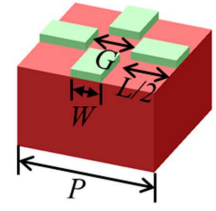

(b)

Figure 1. (a) Schematic of the investigated artificial spin ice array; (b) unit cell of the array as marked in (a) by dashed line. 
magnetization distribution of the RS10 and RS11 states at zero field, for the ASI array with $G=130 \mathrm{~nm}$, are shown in Figure 2(a) and Figure 2(b), respectively. For the RS10 state, the magnetization orients along the $+x$ direction for the horizontal elements, and the magnetization is not a single domain in the vertical elements of the array. In the vertical elements, the magnetization of the left and right part points to the inverse direction, respectively. For the RS11 state, the magnetization orients along the $+y$ direction in the vertical elements and along the $+x$ direction for the horizontal elements of the ASI array.

To investigate the effect of magnetization distribution on the microwave permeability of the ASI lattice, we firstly calculated the complex permeability of lattice with $G=130 \mathrm{~nm}$ at RS10 and RS11 states for zero field as shown in Figure 3. For the RS10 state, the main resonance peak with the resonance frequency of $9.6 \mathrm{GHz}$ can be identified with the uniform resonance mode [10]. For the RS11 state, the resonance peak locates at $9.0 \mathrm{GHz}$. This resonance mode can be attributed to the uniform resonance mode as what is observed for the RS10 state.

The calculated permeability and the resonance frequency as a function of the applied magnetic field strength, for the ASI array with $G=130 \mathrm{~nm}$, are shown in Figure 4. It is observed that both the resonance frequency and the amplitude of the imaginary part of the permeability are strongly dependent on the strength of the applied magnetic field. For the RS10 case as shown in Figure 4(a), the resonance frequency increases from 9.6 to 17.1 $\mathrm{GHz}$ when the applied field increases from 0 to $200 \mathrm{mT}$. While the amplitude of the imaginary part of the permeability decrease from 65.7 to 49.2. For the RS11 case as shown in Figure 4(b), the resonance frequency increases from 9.1 to $16.6 \mathrm{GHz}$ when the applied field increases from 0 to $200 \mathrm{mT}$. While the amplitude of the imaginary part of the permeability decrease from 71.8 to 2.3 . Therefore, the permeability of the ASI array at the

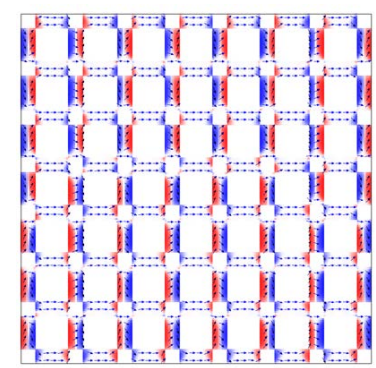

(a)

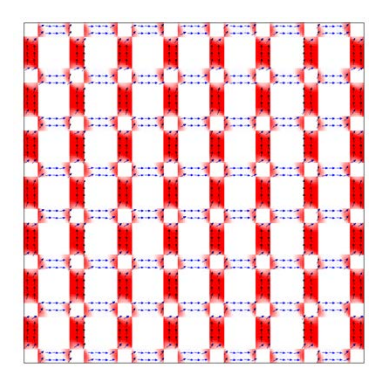

(b)

Figure 2. Magnetization distribution of the ASI lattice in the ground magnetic state with the applied field of $0 \mathrm{mT}$ for (a) RS10 and RS11.

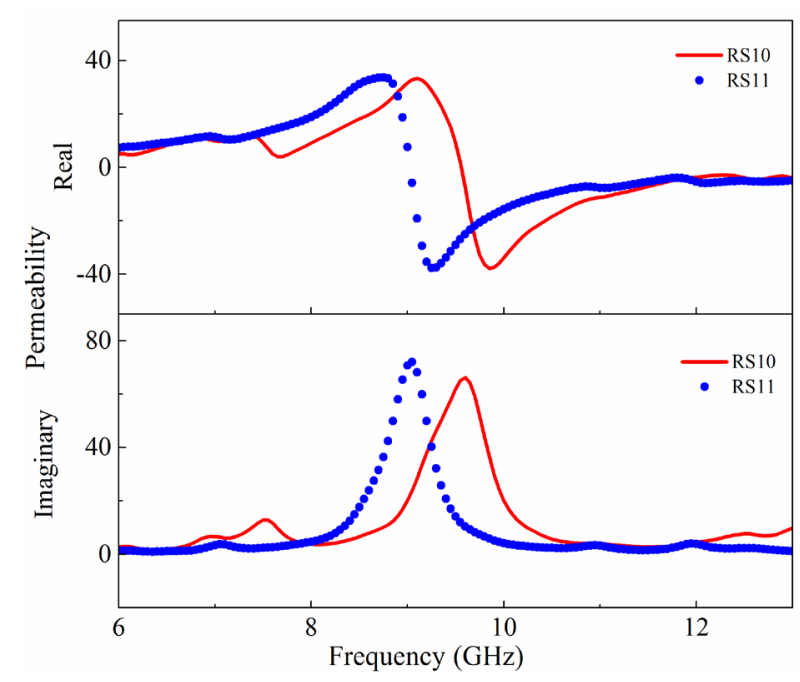

Figure 3. Calculated complex permeability of the ASI array at zero applied magnetic field for the two ground states: the solid line for RS10 and the dotted line for RS11. 
RS11 state is more sensitive to the applied magnetic field with a variation of 69.5 , which is much larger than that of the RS10 state of 16.5 .

To investigate the effect of the gap $G$ on the permeability spectrum of the ASI array, we also calculated the permeability of ASI arrays with various $G$ values at zero magnetic field. As shown in Figure 5, the permeability of the ASI array is observed to be dependent on the gap $G$ values. For the RS10 case, the resonance frequency decreases from 9.6 to $9.0 \mathrm{GHz}$ when the gap $G$ value increases from 130 to $230 \mathrm{~nm}$. While the amplitude of the imaginary part of the permeability increase from 65.8 to 72.6 . For the RS11 case as shown in Figure 5(b), the resonance frequency decreases from 9.0 to $8.8 \mathrm{GHz}$ the gap $G$ value increases from 130 to $230 \mathrm{~nm}$. While the amplitude of the imaginary part of the permeability decreases from 71.8 to 70.8 . Therefore, the permeability of the ASI array at the RS10 state is more sensitive to the variation of the gap between the neighboring elements of the ASI array.

\section{Conclusion}

In summary, the microwave complex permeability of an artificial square spin ice array is investigated using the micromagnetic simulation method. It is observed that the permeability spectrum of the ASI array exhibits different properties depending on the magnetization saturating direction. And the permeability is strongly dependent on the external applied magnetic field and the spacing gap between the comprising elements of the ASI array. Among the two studied RS10 and RS11 cases, the permeability spectrum at the RS11 state is more sensitive to the external applied magnetic field. However, the permeability spectrum at the RS10 state is more sensitive to

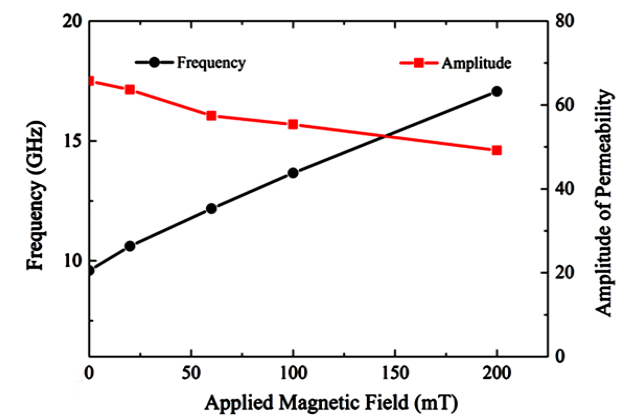

(a)

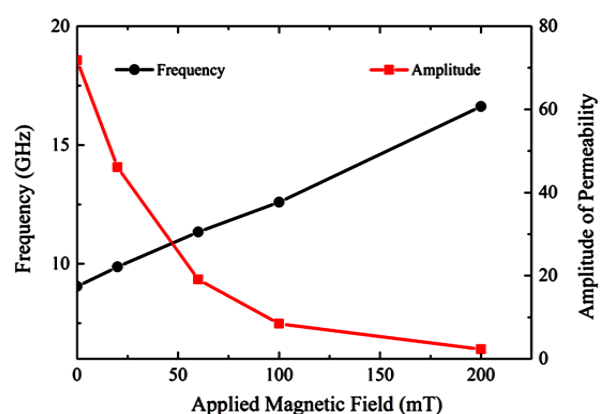

(b)

Figure 4. Magnetic field strength dependence of the resonant frequency of the uniform resonance mode and the amplitude of the imaginary part of the permeability for (a) RS10 and (b) RS11.

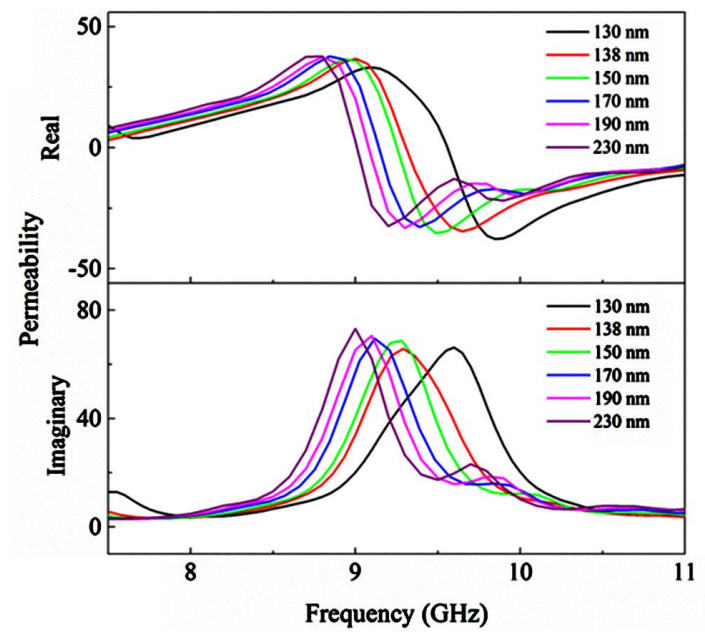

(a)

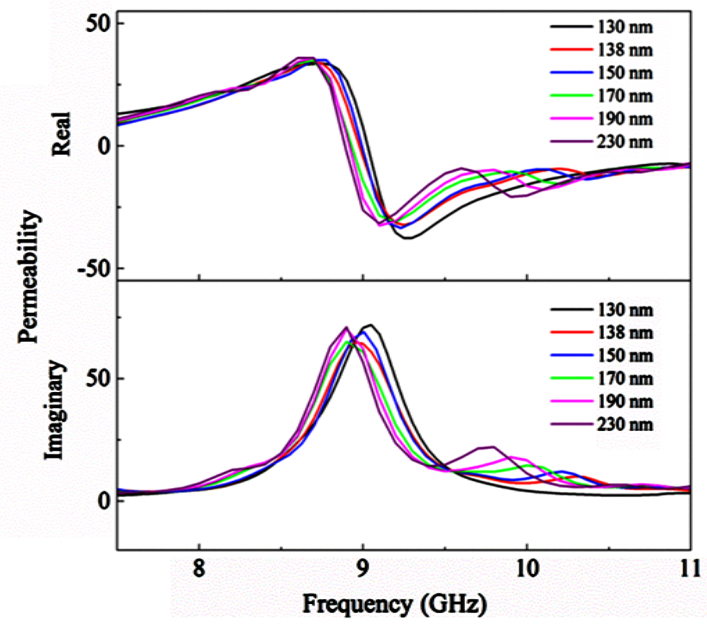

(b)

Figure 5. Gap dependence of the complex permeability of the ASI array at zero applied magnetic field for the ground states: (a) RS10 and (b) RS11. 
the spacing gap between the comprising elements of the ASI array. Our findings would be useful for the investigation of these novel artificial structures and their potential application for the microwave devices, i.e. tunable microwave filters working on different frequencies.

\section{References}

[1] Wang, R.F., Nisoli, C., Freitas, R.S., Li, J., McConville, W., Cooley, B.J., Lund, M.S., Samarth, N., Leighton, C., Crespi, V.H. and Schiffer, P. (2006) Artificial "Spin Ice" in a Geometrically Frustrated Lattice of Nanoscale Ferromagnetic Islands. Nature, 439, 303-306. http://doi:10.1038/nature04447

[2] Heyderman, L.J. and Stamps, R.L. (2013) Artificial Ferroic Systems: Novel Functionality from Structure, Interactions and Dynamics. Journal of Physics: Condensed Matter, 25, 363201. http://doi:10.1088/0953-8984/25/36/363201

[3] Nisoli, C., Moessner, R. and Schiffer, P. (2013) Colloquium: Artificial Spin Ice: Designing and Imaging Magnetic Frustration. Reviews of Modern Physics, 85, 1473-1490. http://dx.doi.org/10.1103/RevModPhys.85.1473

[4] Tanaka, M., Saitoh, E., Miyajima, H., Yamaoka, T. and Iye, Y. (2006) Magnetic Interactions in a Ferromagnetic Honeycomb Nanoscale Network. Physical Review B, 73, 052411. http://dx.doi.org/10.1103/PhysRevB.73.052411

[5] Donahue, M.J. and Porter, D.G. OOMMF User’s Guide, Version 1.0. http://math.nist.gov/oommf/

[6] Gérardin, O., Ben Youssef, J., Le Gall, H., Vukadinovic, N., Jacquart, P.M. and Donahue, M.J. (2000) Micromagneics of the Dynamic Susceptibility for Coupled Permalloy Stripes. Journal of Applied Physics, 88, 5899-5903. http://dx.doi.org.libproxy1.nus.edu.sg/10.1063/1.1320011

[7] Dao, N., Donahue, M.J., Dumitru, I., Spinu, L., Whittenburg, S.L. and Lodder, J.C. (2004) Dynamic Susceptibility of Nanopillars. Nanotechnology, 15, S634-S638. http://doi:10.1088/0957-4484/15/10/022

[8] Ma, F.S., Lim, H.S., Wang, Z.K., Piramanayagam, S.N., Ng, S.C. and Kuok, M.H. (2011) Micromagnetic Study of Spin Wave Propagation in Bicomponent Magnonic Crystal Waveguides. Applied Physics Letters, 98, 153107. http://dx.doi.org.libproxy1.nus.edu.sg/10.1063/1.3579531

[9] Ma, F.S., Lim, H.S., Wang, Z.K., Piramanayagam, S.N., Ng, S.C. and Kuok, M.H. (2012) Band Structures of Exchange Spin Waves in One-Dimensional Bi-Component Magnonic Crystals. Journal of Applied Physics, 111, 064326. http://dx.doi.org.libproxy1.nus.edu.sg/10.1063/1.3698617

[10] Gérardin, O., Le Gall, H., Donahue, M.J. and Vukadinovic, N. (2001) Micromagnetic Calculation of the High Frequency Dynamics of Nano-Size Rectangular Ferromagnetic Stripes. Journal of Applied Physics, 89, 7012. http://dx.doi.org.libproxy1.nus.edu.sg/10.1063/1.1360390 
Scientific Research Publishing (SCIRP) is one of the largest Open Access journal publishers. It is currently publishing more than 200 open access, online, peer-reviewed journals covering a wide range of academic disciplines. SCIRP serves the worldwide academic communities and contributes to the progress and application of science with its publication.

Other selected journals from SCIRP are listed as below. Submit your manuscript to us via either submit@scirp.org or Online Submission Portal.
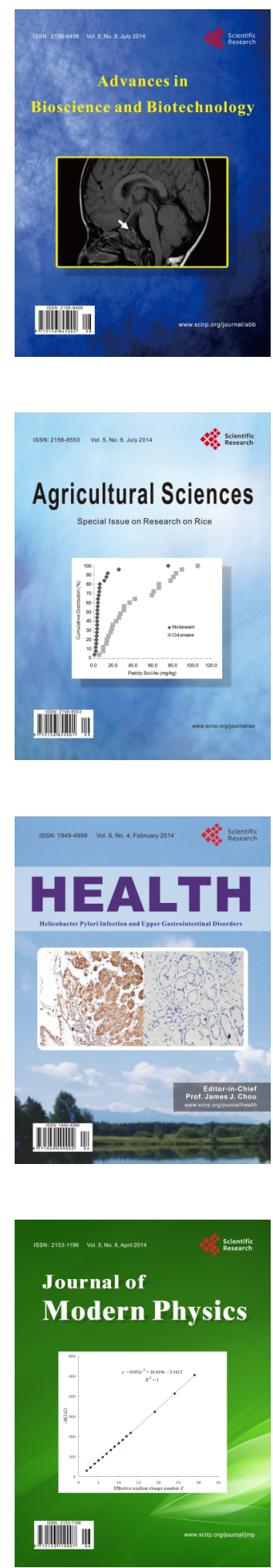
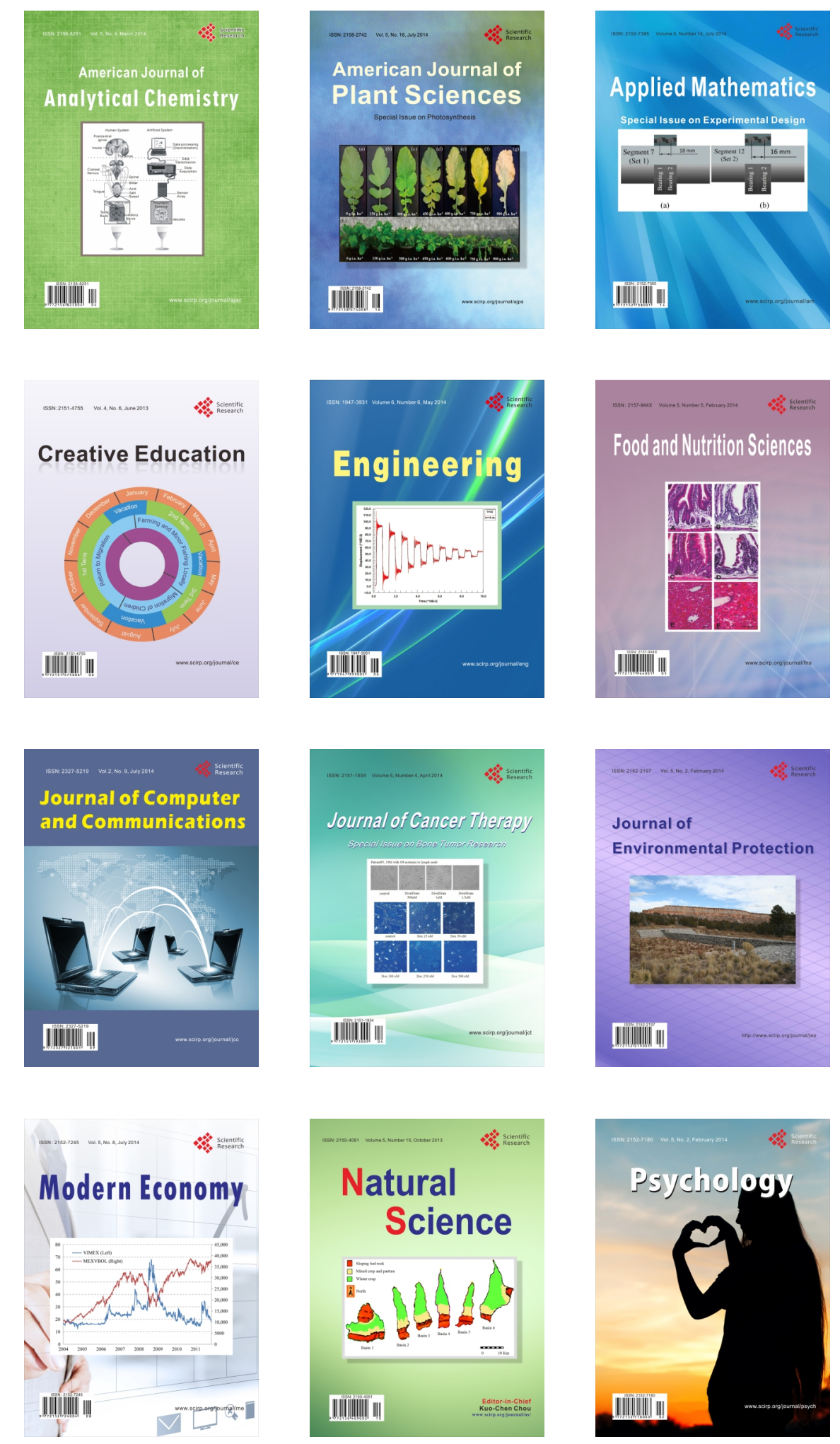